\title{
Improving the Prescription of Oral Anticoagulants in Atrial Fibrillation: A Systematic Review
}

\author{
Ruth V. Pritchett ${ }^{1}$ Danai Bem ${ }^{1}$ Grace M. Turner ${ }^{1}$ \\ Rebecca Fellows ${ }^{1}$ Deirdre A. Lane ${ }^{2,3}$ Kate Jolly ${ }^{1}$ \\ ${ }^{1}$ Institute of Applied Health Research, University of Birmingham, \\ Birmingham, United Kingdom \\ 2 Institute of Cardiovascular Sciences, University of Birmingham, \\ Birmingham, United Kingdom \\ ${ }^{3}$ Aalborg Thrombosis Research Unit, Department of Clinical \\ Medicine, Aalborg University, Aalborg, Denmark \\ Thromb Haemost 2019;119:294-307.
}

\author{
G. Neil Thomas ${ }^{1}$ Joanne L. Clarke ${ }^{1}$
}

\begin{abstract}
Address for correspondence Ruth V. Pritchett, BMedSc, PhD, Institute of Applied Health Research, University of Birmingham, Birmingham, United Kingdom (e-mail: r.v.pritchett@bham.ac.uk).
\end{abstract}

\begin{abstract}
Objective Oral anticoagulant (OAC) prescription for stroke prevention in atrial fibrillation (AF) patients frequently does not follow current guidelines, with underuse in patients at high risk of stroke and substantial overuse in those at low risk. This review aims to systematically evaluate the effectiveness of interventions to improve appropriate OAC prescription in eligible AF patients for stroke prevention.

Methods Systematic review of controlled and uncontrolled studies published up to July 2017 with interventions designed to improve appropriate OAC prescription for stroke prevention in eligible AF patients (according to risk assessment tool or guidelines). Categorization of intervention types was pre-specified. The main outcome was change in proportion of eligible AF patients prescribed OACs for stroke prevention.

Results Twenty studies conducted in 392 settings were included (cluster randomized controlled trials, controlled trials and uncontrolled before-after designs; $n=29,868$ patients at baseline). Fifteen studies reported significant improvements in appropriate prescription of OACs in AF patients. All interventions with a persuasive element (8/8); all studies targeting health care professional (HCP) education or guideline/protocol implementation (7/7); and all medical care programs (4/4) achieved significant increases in appropriate OAC prescription. Computerized decision support interventions (3/5) and reviews of prescribing (2/4) were less likely to report significant improvements in

Keywords

- atrial fibrillation

- oral anticoagulation

- systematic review

- intervention

- stroke prevention appropriate OAC prescription.

Conclusion Interventions designed to improve appropriate prescription of OACs in eligible AF patients for stroke prevention can be effective. Successful approaches include education of HCPs; implementation of local guidelines; interdisciplinary medical care programs educating both $\mathrm{HCPs}$ and patients and persuasive interventions utilizing peergroup experts. Protocol registration: PROSPERO (CRD42016039654).
\end{abstract}

\section{Introduction}

Atrial fibrillation (AF) currently affects over 33 million people worldwide with increasing prevalence in the United Kingdom and globally. ${ }^{1,2}$ Current national and international guidance recommends that female AF patients with a $\mathrm{CHA}_{2} \mathrm{DS}_{2}$-VASc score of $\geq 2$ and male patients with a score of $\geq 1$ should be offered oral anticoagulation (OAC) including vitamin $\mathrm{K}$ received

September 3, 2018

accepted after revision

November 6, 2018
DOI https://doi.org/

10.1055/s-0038-1676835.

ISSN 0340-6245. (c) 2019 Georg Thieme Verlag KG Stuttgart · New York
License terms

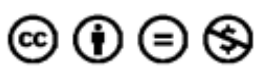


antagonists (VKAs) and non-VKA oral anticoagulants (NOACs) to reduce risk of stroke ${ }^{3-5}$ (taking bleeding into account using the HAS-BLED score ${ }^{6}$ ). Over the last decade, rates of OAC use for stroke prevention in newly diagnosed AF patients have steadily increased to $71 \%$ worldwide ${ }^{7}$; reaching around $80 \%$ in Europe and the United States. ${ }^{7,8}$ However, there is still substantial room for improvement in appropriate, guideline-adherent, OAC prescribing. Worldwide, around half of all newly diagnosed AF patients with a $\mathrm{CHA}_{2} \mathrm{DS}_{2}$-VASc score of 0 (low risk) are prescribed OACs contrary to guideline advice, putting them at unnecessary risk of haemorrhage. ${ }^{7}$ Conversely, of all high-risk U.K. patients with a $\mathrm{CHA}_{2} \mathrm{DS}_{2}$-VASc score of $\geq 2$ only $68 \%$ are receiving OACs, falling to $40 \%$ in India and $31 \%$ in China, ${ }^{7}$ exposing many to a risk of stroke. Under-prescription of OACs in patients aged 65 years and above has also been reported across Europe. ${ }^{9}$

Research has explored possible reasons for under-prescription of OACs in AF patients, with general practitioners (GPs) reporting feeling responsible for haemorrhages in anticoagulated patients. ${ }^{10}$ Patients' co-morbidities and concerns about their ability to achieve adequate time in therapeutic range may create barriers to the prescription of VKAs. ${ }^{10}$ Health care professionals (HCPs) may be especially reluctant to prescribe OACs to older adults due to a perceived increased likelihood of falls and subsequent haemorrhage; however, research suggests that stroke risk is much greater in older adults with $\mathrm{AF}$, making anticoagulation more vital. ${ }^{10}$

Interventions have attempted to improve HCPs' adherence to guidelines in prescribing OACs to AF patients, ${ }^{11}$ but there is no clear evidence regarding which intervention design and theoretical framework is most effective. This article presents the first review to systematically evaluate the effectiveness of interventions with any comparator designed to improve appropriate prescription of OACs in eligible AF patients for stroke prevention.

\section{Methods}

\section{Protocol and Registration}

The systematic methodology of this review was based on the Cochrane Collaboration handbook ${ }^{12}$ and the Preferred Reporting Items for Systematic Reviews and Meta-Analyses statement. ${ }^{13}$ The review protocol was registered on PROSPERO prior to conducting literature searches (CRD42016039654).

\section{Eligibility Criteria}

Quantitative studies reporting interventions designed to increase the rate of prescription of OACs for stroke prevention in AF patients eligible for OAC treatment, or to improve adherence to guidelines, were considered for inclusion. Patients eligible for $O A C$ treatment were defined as $\mathrm{CHA}_{2} \mathrm{DS}_{2}$-VASc score of $\geq 2$ if female, $\geq 1$ if male, or equivalent risk stratification tool, or stated as eligible according to guidelines. Eligible study designs included controlled studies (randomized or non-randomized, prospective or retrospective, concomitant or historical control) and uncontrolled before-after studies with any comparator. Studies focusing on any HCP prescriber in any health care setting were eligible. Interventions designed to improve both HCPs' prescription of and patients' uptake/adherence to OACs were eligible provided the rate of OAC prescription was reported. Interventions were categorized as 'Persuasive' and/or 'Educational and informational', and/or 'Action and monitoring', according to Johnson and May's intervention types for behavioural change in HCPs. ${ }^{14}$ Studies with an outcome describing change in the proportion of AF patients eligible for OAC treatment who were prescribed OACs by HCPs for stroke prevention were included (or change in the proportion of such patients taking OACs, if the intervention was aimed only at improving HCPs' prescription and not patient uptake).

Studies including patients with a $\mathrm{CHA}_{2} \mathrm{DS}_{2}$-VASc score of $<2$ if female or $<1$ if male (or ineligible for OACs on an equivalent risk stratification tool or according to guidelines) were excluded from this review. Interventions focusing only on the patient, or studies whose outcome was the proportion of patients eligible for OACs who were taking OACs, where the intervention was aimed at both the patient and the health professional were excluded. Qualitative studies and case series were also excluded from this review.

\section{Literature Searches}

Searches were performed with no language/publication date restrictions from inception to June 2016 in the bibliographic databases, including MEDLINE and MEDLINE in Process, EMBASE, PsycINFO, CINAHL and The Cochrane Library (CDSR, DARE and CENTRAL); Science Citation Index (Web of Science) for citation searching; World Health Organization International Clinical Trials Registry Platform and Clinical Trials.gov for trials in progress; Conference Proceedings Citation Index; Open Gray; and the Health Management Information Consortium for grey literature and bibliographies of relevant articles. A combination of text words and index terms related to the condition (AF), the therapy $(\mathrm{OAC})$ and the intervention (interventions to increase appropriate OAC prescription) were utilized (-Supplementary Fig. S1, available in the online version). A search update was performed up to July 2017 using the limits 'therapy' and 'best balance' to concentrate the search on OAC therapy, locating relevant papers while avoiding irrelevant material.

\section{Study Selection Process}

Search results were exported to EndNote V.X7.4 (Thomson Reuters, New York, New York, United States) and duplicates removed. Titles and abstracts were read for relevance and fulltext versions of potentially eligible publications obtained. Non-English language articles were translated. A standardized, pre-determined, study criteria form was applied to all full-text documents, with ineligible publications and reasons for ineligibility recorded (- Supplementary Table S1, available in the online version).

\section{Data Extraction and Quality Assessment}

Data were extracted using a standardized, piloted data extraction form. Discrepancies were compared with original data. 
Information on following characteristics was extracted: study design, health care setting, AF type and risk stratification, intervention/comparator arms, intervention-deliverer, target population and findings. The theoretical focus of the intervention was determined based on the categories suggested by Johnson and May: persuasive interventions (marketing, mass media, local consensus processes, local opinion leaders); educational and informational interventions (educational materials and meetings); and action and monitoring interventions (audit and feedback, reminders). ${ }^{14}$

Risk of bias assessment was tailored to different study designs. The Cochrane Collaboration's risk of bias tool was used to quality assess randomized controlled studies ${ }^{15}$ (-Supplementary Fig. S2, available in the online version); an adjusted version was used for non-randomized controlled studies (-Supplementary Fig. S3, available in the online version). The National Institutes of Health (NIH) Regional Heart, Lung and Blood Institute Quality Assessment Tool for observational, cohort and cross-sectional studies was used to assess risk of bias in cross-sectional studies ${ }^{16}$ (- Supplementary Fig. S4, available in the online version); and an adapted version of the NIH Quality Assessment Tool for before-after studies with no control group was used to assess risk of bias in uncontrolled before-after studies ${ }^{16}$ ( - Supplementary Fig. $\mathbf{5 5}$, available in the online version).

All study selection, data extraction and quality assessment processes were conducted independently and in duplicate; discrepancies were resolved by an additional reviewer.

\section{Data Synthesis}

As study designs were heterogeneous, a narrative synthesis was conducted. Tables of characteristics were created for eligible controlled and uncontrolled studies ( - Tables 1 and 2; further details in - Supplementary Table $\mathbf{5 2}$, available in the online version). To facilitate comparison, data were summarized in a standardized way. Results were considered by different characteristics and risk of bias to elicit systematic differences ( $\boldsymbol{- T a b l e ~} \mathbf{3}$ ). It was not possible to formally assess possible publication bias.

\section{Results}

This review identified 12,807 records, of which 72 were assessed for full eligibility; of these, 52 did not meet eligibility criteria (-Supplementary Table S1, available in the online version). Twenty studies were included in this review and informed the analysis (-Fig. 1). Protocol papers of four on-going studies were located. ${ }^{17-20}$

\section{Study Characteristics}

The 20 included studies were conducted at 392 individual health care settings (including GP surgeries and secondary/ tertiary care hospitals) (-Tables $\mathbf{1}$ and $\mathbf{2}$ ). At baseline, 29,868 patients were included; 11,105 in controlled and 18,763 in uncontrolled studies. Study designs included 3 cluster randomized controlled trials (RCTs), ${ }^{21-23} 5$ controlled studies, ${ }^{24-28} 1$ cross-sectional study ${ }^{29}$ and 11 uncontrolled before-after studies. ${ }^{30-40}$

\section{Intervention Design}

Of the 20 studies, one provided an educational intervention with an expert panel, ${ }^{40}$ three were based on implementation of local or national clinical guidelines, ${ }^{28,32,34}$ two implemented a clinical protocol ${ }^{35,37}$ and one comprised a multicomponent intervention (education, decision support tool, performance feedback). ${ }^{38}$ Three were based on a review of current prescribing, ${ }^{25,33,36}$ and one incorporated a review of prescribing and the introduction of a clinical protocol. ${ }^{31}$ Five integrated computerized decision support or risk assessment tools into their systems ${ }^{21-23,27,30}$ and four were based on medical care programs offering HCP training and HCPpatient consultations. ${ }^{24,26,29,39}$ To improve OAC prescription, six interventions used action and monitoring techniques, ${ }^{21-24,26,30}$ six combined education and interventional techniques with action and monitoring, ${ }^{25,27,32,33,37,39}$ four attempted to use persuasion plus educational and interventional techniques, ${ }^{28,35,36,40}$ one used persuasion plus action and monitoring ${ }^{31}$ and three were multifaceted, using persuasive, educational and interventional and action and monitoring techniques. ${ }^{29,34,38}$

Interventions were delivered by a range of professionals, with eight delivered by either researchers or a combination of researchers and HCPs such as primary, secondary and tertiary care clinicians, nurses, pharmacists, allied HCPs and software suppliers. ${ }^{21-23,25,27,28,33,37}$ Ten interventions were delivered by HCPs only, including secondary care clinicians, GPs, primary care teams, clinical pharmacists, allied and nursing HCPs and local clinical stakeholders. Two interventions had unspecified deliverers. ${ }^{24,32}$ Five interventions were aimed at improving OAC prescribing among GPs, ${ }^{21-23,28,38}$ three at hospital physicians, ${ }^{24,25,27}$ one at cardiologists, ${ }^{26}$ four at interdisciplinary primary care teams, $29,31,37,39$ one at an interdisciplinary hospital team, ${ }^{40}$ two at hospital prescribers ${ }^{30,32}$ and four at hospital clinicians and GPs. ${ }^{33-36}$

\section{Follow-Up}

Of the 12 uncontrolled studies, only Bajorek et al provided follow-up beyond the post-intervention data (3- and 6month follow-up). ${ }^{33}$ of the three RCTs, one provided a maximum follow-up of 11 months ${ }^{22}$ and two provided data at 12 months. ${ }^{21,23}$ Four of the five controlled trials provided data from historical control groups that commenced 4 months, ${ }^{25} 1^{27,28}$ year and 3 years ${ }^{26}$ before the intervention cohorts, respectively. The final controlled study reported an average follow-up length of 25 months $^{24}$ (-Supplementary Table S2, available in the online version).

\section{Fidelity}

Measures assessing the fidelity of the interventions to the intended method were provided by four studies. $22-24,40$ Hsieh et al reported that the proportion of HCPs completing five stroke quality measures increased from $75 \%$ before the intervention to $86 \%$ during the intervention. ${ }^{40}$ Arts et al monitored the triggers for notifications and the usage of their notification system, reporting 3,848 notifications, of which $188(5 \%)$ were clicked on for further information, 76 were 







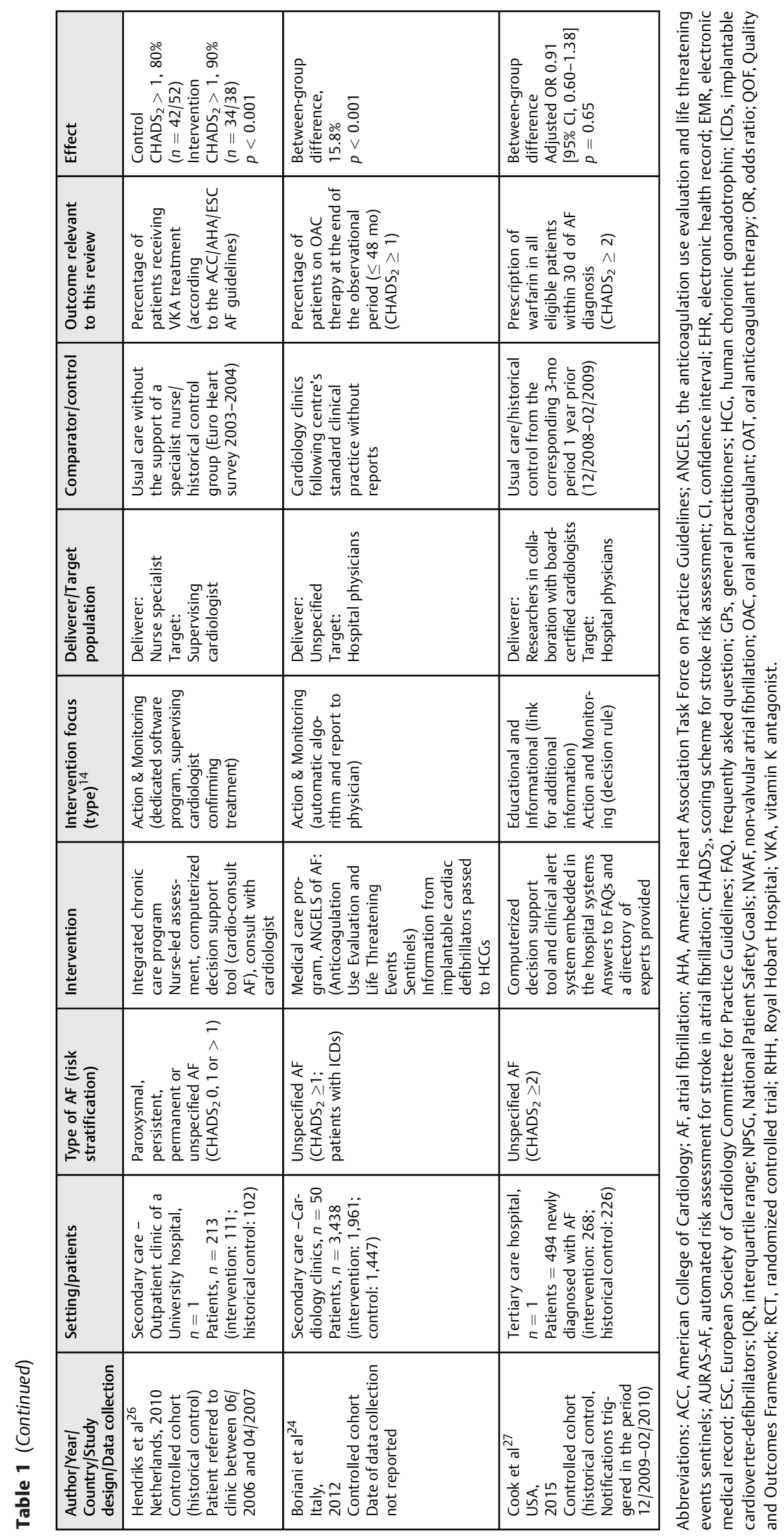









\begin{tabular}{|c|c|c|c|c|c|}
\hline 志 & 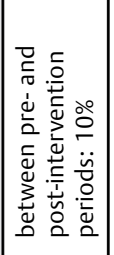 & 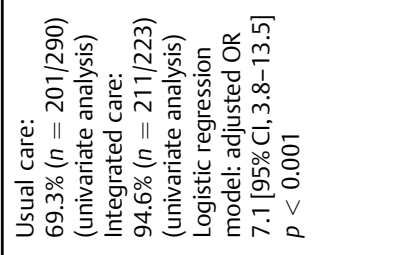 & 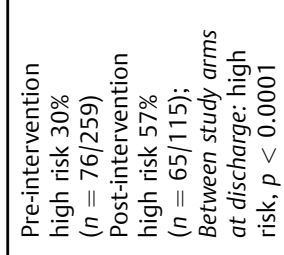 &  & 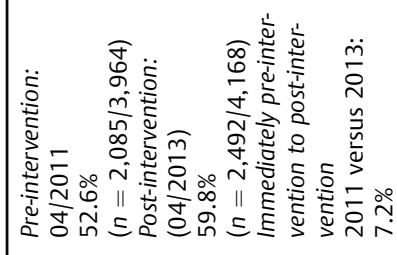 \\
\hline 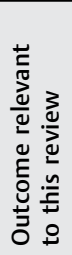 & &  & 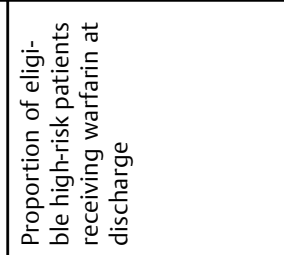 & 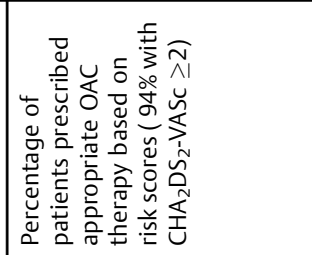 & 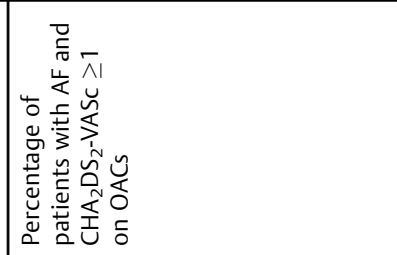 \\
\hline & & 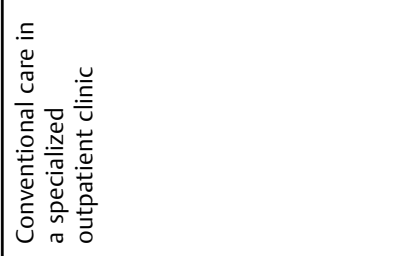 & 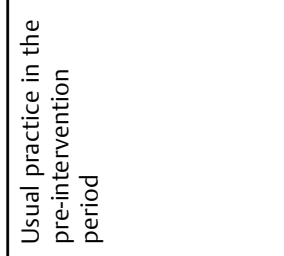 & 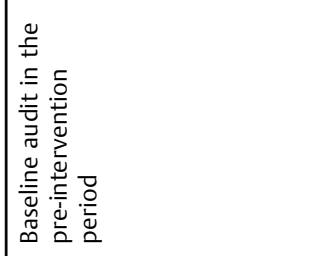 &  \\
\hline 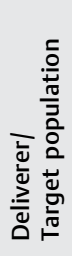 & &  & 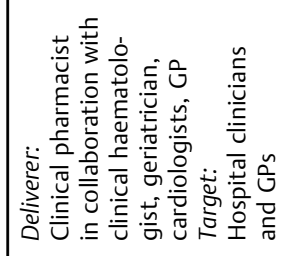 & 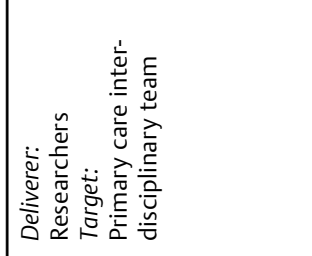 &  \\
\hline  & & 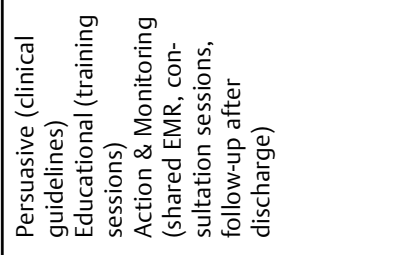 &  & 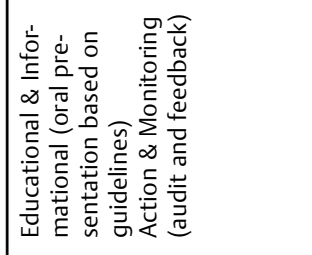 &  \\
\hline  &  & 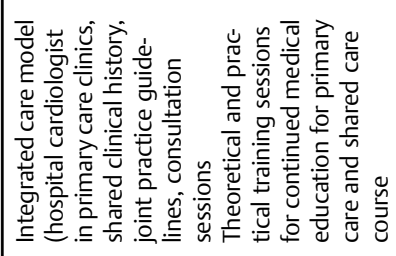 & 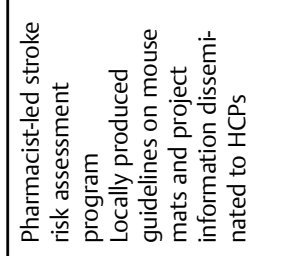 & 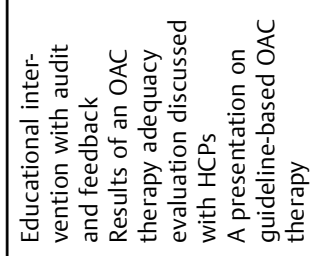 & 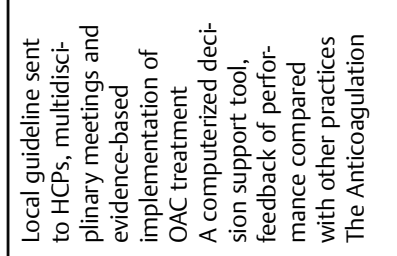 \\
\hline  & &  & 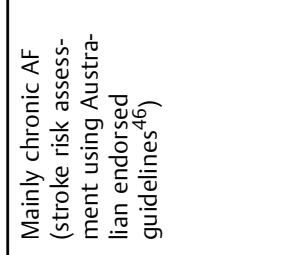 &  & 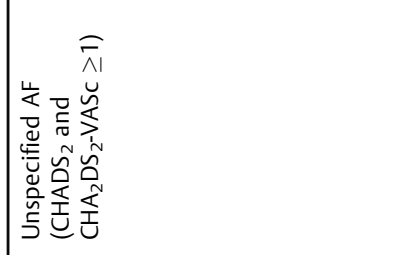 \\
\hline  & & 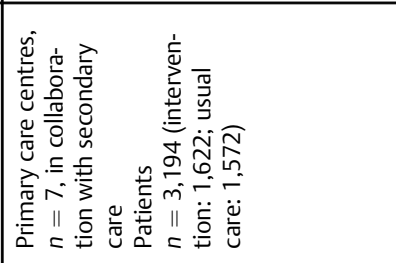 &  &  & 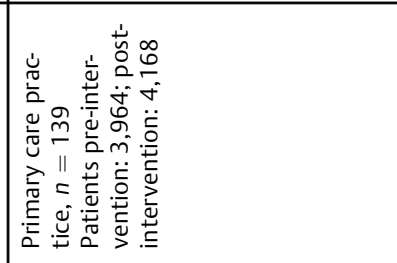 \\
\hline 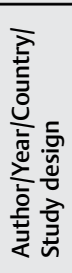 & & 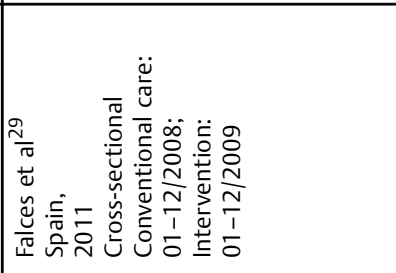 & 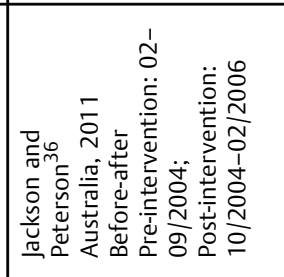 & 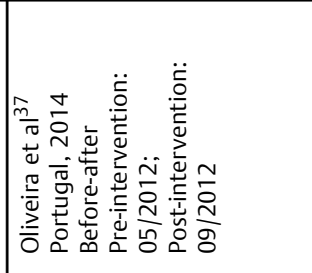 &  \\
\hline
\end{tabular}







Table 3 Effectiveness of interventions by study characteristics

\begin{tabular}{|c|c|c|c|}
\hline \multirow[t]{2}{*}{ Characteristic } & \multirow[t]{2}{*}{ Category } & \multicolumn{2}{|c|}{$\begin{array}{l}\text { Change in appropriate OAC prescription/ } \\
\text { use (total } n=20 \text { studies) }\end{array}$} \\
\hline & & Significant increase & No significant change \\
\hline \multirow{2}{*}{$\begin{array}{l}\text { Date } \\
\text { published }\end{array}$} & Pre-2010 & $5 / 7$ & $2 / 7$ \\
\hline & 2010 and after & $10 / 13$ & $3 / 13$ \\
\hline \multirow[t]{2}{*}{ Country } & EU & $10 / 12$ & $2 / 12$ \\
\hline & Non-EU & $5 / 8$ & $3 / 8$ \\
\hline \multirow[t]{2}{*}{ Study design } & Uncontrolled or historical control & $13 / 16$ & $3 / 16$ \\
\hline & Concurrent control & $2 / 4$ & $2 / 4$ \\
\hline \multirow[t]{3}{*}{ Setting } & Primary care & $7 / 9$ & $2 / 9$ \\
\hline & Secondary care & $5 / 7$ & $2 / 7$ \\
\hline & Tertiary care & $3 / 4$ & $1 / 4$ \\
\hline \multirow{3}{*}{$\begin{array}{l}\text { Date of data } \\
\text { collection }\end{array}$} & Commenced pre-2010 & $10 / 12$ & $2 / 12$ \\
\hline & Commenced 2010 and after & $4 / 6$ & $2 / 6$ \\
\hline & Unknown & $1 / 2$ & $1 / 2$ \\
\hline \multirow[t]{3}{*}{ Type of AF } & All/unspecified & $10 / 15$ & $5 / 15$ \\
\hline & Chronic & $3 / 3$ & $0 / 3$ \\
\hline & Non-valvular & $2 / 2$ & $0 / 2$ \\
\hline \multirow[t]{3}{*}{ Severity of AF } & $\begin{array}{l}\mathrm{CHADS}_{2} \text { or } \mathrm{CHA}_{2} \mathrm{DS}_{2} \text {-VASc } \geq 2 \text { or } \geq 75 \text { years old } \\
\text { or high-risk according to guidelines }\end{array}$ & $5 / 8$ & $3 / 8$ \\
\hline & $\begin{array}{l}\mathrm{CHADS}_{2} \text { and } \mathrm{CHA}_{2} \mathrm{DS}_{2} \text {-VASc } \geq 1 \text { or } \geq 65 \text { years old, } \\
\text { or moderate- and high-risk according to guidelines }\end{array}$ & $6 / 7$ & $1 / 7$ \\
\hline & Unknown/any severity & $4 / 5$ & $1 / 5$ \\
\hline \multirow[t]{4}{*}{ Intervention } & Computerized risk assessment tool & $2 / 5$ & $3 / 5$ \\
\hline & Education/guidelines/protocol & $7 / 7$ & $0 / 7$ \\
\hline & Medical care program & $4 / 4$ & $0 / 4$ \\
\hline & Review of prescribing & $2 / 4$ & $2 / 4$ \\
\hline \multirow{5}{*}{$\begin{array}{l}\text { Intervention } \\
\text { focus }\end{array}$} & Action and monitoring & $4 / 6$ & $2 / 6$ \\
\hline & Educational and interventional; Action and monitoring & $3 / 6$ & $3 / 6$ \\
\hline & Educational and interventional; Persuasive & $4 / 4$ & $0 / 4$ \\
\hline & Persuasive; Action and monitoring & $1 / 1$ & $0 / 1$ \\
\hline & $\begin{array}{l}\text { Educational and interventional; } \\
\text { Persuasive; Action and monitoring }\end{array}$ & $3 / 3$ & $0 / 3$ \\
\hline \multirow{3}{*}{$\begin{array}{l}\text { Intervention } \\
\text { deliverer }\end{array}$} & Health care professional (HCP) & $9 / 10$ & $1 / 10$ \\
\hline & Researchers (and/or HCPs) & $4 / 8$ & $4 / 8$ \\
\hline & Unspecified & $2 / 2$ & $0 / 2$ \\
\hline \multirow{3}{*}{$\begin{array}{l}\text { Target } \\
\text { population }\end{array}$} & GPs & $3 / 5$ & $2 / 5$ \\
\hline & Primary care interdisciplinary team & $4 / 4$ & $0 / 4$ \\
\hline & $\begin{array}{l}\text { Secondary care/Secondary and primary care/ } \\
\text { Secondary care interdisciplinary team }\end{array}$ & $8 / 11$ & $3 / 11$ \\
\hline \multirow[t]{2}{*}{ Comparator } & Control health care settings (usual care) & $3 / 5$ & $2 / 5$ \\
\hline & $\begin{array}{l}\text { Usual care at the same site prior to the } \\
\text { intervention or a historical control }\end{array}$ & $12 / 15$ & $3 / 15$ \\
\hline
\end{tabular}

Abbreviations: AF, atrial fibrillation; EU, European Union; GP, general practitioner; OAC, oral anticoagulant. 


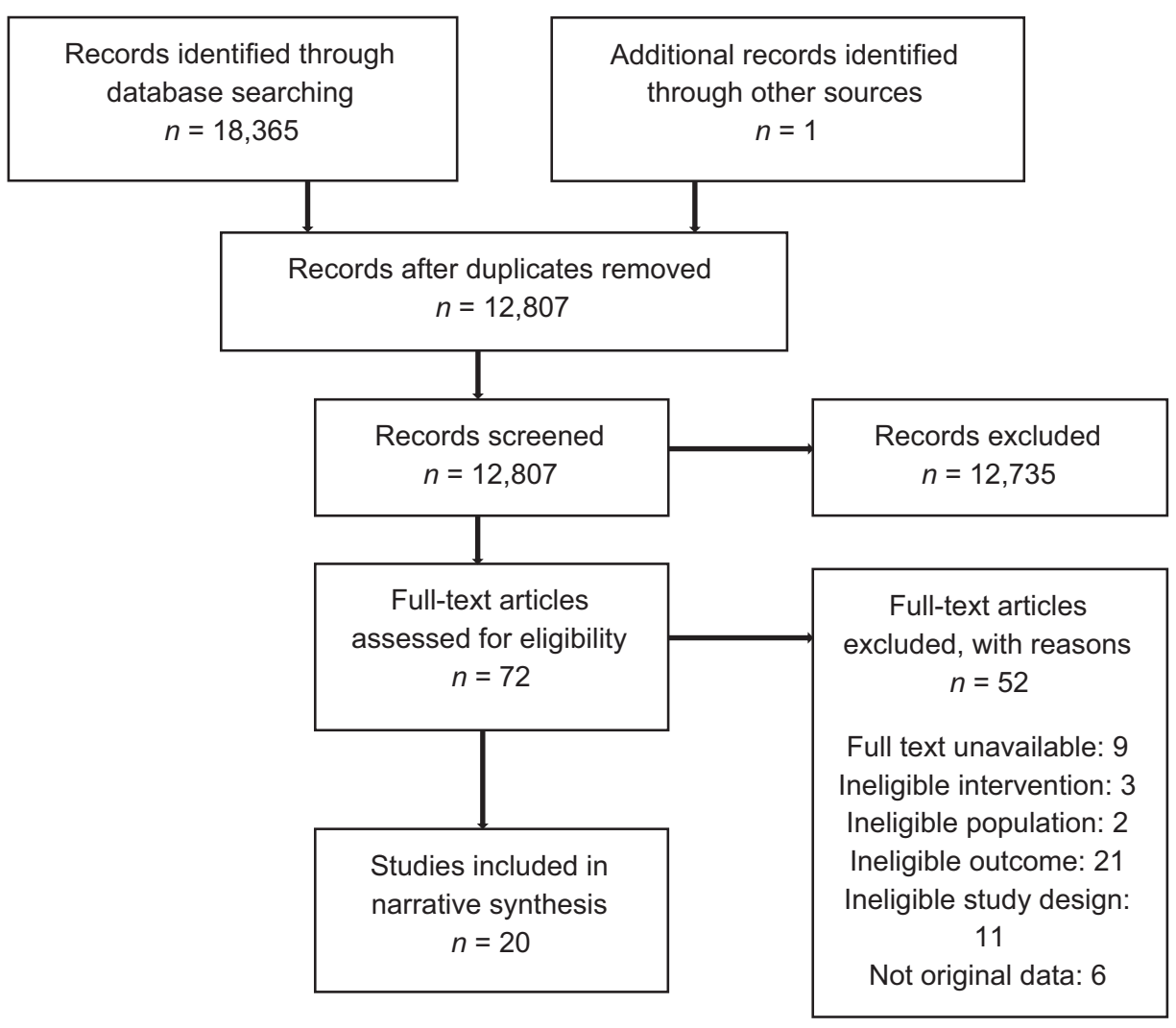

Fig. 1 Preferred Reporting Items for Systematic Reviews and Meta-Analyses (PRISMA) statement flowchart.

actively responded to, of which 44 (58\%) were dismissed, and $32(42 \%)$ were accepted, indicating the advice would be followed. ${ }^{22}$ Boriani et al reported that the ANGELS of AF reports were the specific trigger for prescribing OACs to 22 (10.5\%) patients of the 209 not already receiving OACs. ${ }^{24}$ Holt et al obtained data from $12 / 23$ intervention practices on HCPs' responses to screen prompts and their invitations to patients to discuss OAC prescription ${ }^{23} ; 466$ patients were identified by the system as eligible at baseline, 159 (34\%) of these were confirmed as eligible by HCPs and 35 (22\%) of these were prescribed OACs. ${ }^{23}$ The mean proportion of those originally identified as eligible who converted to OACs was $4.2 \%^{23}$

\section{Study Quality}

The three cluster RCTs ${ }^{21-23}$ were at low risk of many sources of bias; however, one RCT did not report control group data at follow-up. ${ }^{21}$ One common potential source of performance bias was HCPs being aware of their group allocation. Of the five non-randomized controlled studies, ${ }^{24-28}$ three used historical controls $^{25-27}$ introducing a risk of bias due to changes in guidance and prescribing behaviours over time. In controlled studies, sample size calculations were either absent ${ }^{26,28}$ or their suitability unclear, ${ }^{24,25,27}$ raising the possibility of nonsignificant results due to insufficient sample sizes (ranging from 213 to 5,339 patients); blinding of outcome assessments was also poorly described in three studies. ${ }^{24,26,28}$ The crosssectional study also lacked a sample size calculation. ${ }^{29}$ The before-after studies ${ }^{30-40}$ were generally at low risk of bias according to the adapted version of the NIH assessment tool used ${ }^{16}$ however, in 7 of the 11 studies the population may not have been representative of those who would be eligible for the intervention ${ }^{30,33,34,36-38,40}$ due to differences between the study population and the general population in characteristics such as age, ethnicity, deprivation level or co-morbidities (-Supplementary Figs. S2-S5, available in the online version).

\section{Effectiveness}

Of the 20 studies, 13 reported a significant increase in appropriate OAC prescription/use post-intervention ${ }^{21,24,26,28-32}$ $34,36,38-40$; two reported an increase, though significance was unclear, ${ }^{35,37}$ and five reported no significant effect on appropriate OAC prescription/use 22,23,25,27,33 (- Tables 1 and 2, further details in - Supplementary Table S2, available in the online version).

A greater proportion of non-significant results were reported among more effectively controlled study designs, such as cluster RCTs and studies with a concurrent comparator (2/4), than uncontrolled studies or those with historical controls (3/16). Studies conducted with high stroke-risk patients were less likely to report significant findings (5/8) than those with patients at moderate-to-high $(6 / 7)$ or unknown risk of stroke (4/5). Non-significant results were more frequently reported by studies with interventions based on computerized decision support 
tools (3/5) and reviews of prescribing (2/4); with all studies based on the education of HCPs or guideline/protocol implementation (7/7) and medical care programs offering training to the HCP and HCP-patient consultations (4/4) reporting significant improvements in appropriate OAC prescription. Those interventions based on action and monitoring techniques or both action and monitoring and education and intervention (of which 5/12 were computerized decision support tools) were the least likely to report significant improvements in appropriate OAC prescription (7/12). Interventions with a persuasive element were the most likely to report significant improvements in appropriate OAC prescription: $8 / 8$ versus $7 / 12$ studies with no persuasive element.

Interventions partly delivered by researchers, were less likely to report significant improvements in OAC prescription (4/8) than those delivered by HCPs (9/10); however, $5 / 8$ of the researcher-delivered interventions were based on computerized decision support models, the majority of which reported non-significant results (3/5). None of the interventions delivered by researchers contained a persuasive element-a noteworthy characteristic of effective studies-with $8 / 8$ interventions with a persuasive element reporting significant improvements in appropriate OAC prescription (-Table 3 ).

\section{Clinical Outcomes}

Clinical outcomes such as the rates of stroke, transient ischaemic attack (TIA), embolism and haemorrhage following the intervention were only provided by two studies. ${ }^{24,41}$ Boriani et al reported no difference in the annual embolic event rate in the intervention compared with control group patients $(p=0.64) .{ }^{24}$ Holt et al reported a significantly higher median (interquartile range) incidence of thromboembolic events per 1,000 patients at 6 months in the intervention compared with the control group (10.3 [0, 16.3 vs. $0[0,7.75]])(p=0.03)$; there was no difference at 12-month follow-up. ${ }^{23}$ Haemorrhagic events were non-significantly higher in the control group compared with the intervention group at 6 and 12 months. ${ }^{23}$

\section{Discussion}

This review examined the effectiveness and characteristics of studies designed to increase appropriate OAC prescription in eligible AF patients for stroke prevention. Despite published reviews focusing on improving patient uptake of OACs, ${ }^{42,43}$ this review represents the first rigorously conducted systematic evaluation of interventions to improve HCPs' prescribing behaviours in this field. Research published to date represents a wide variety of study designs, settings, interventions and theoretical approaches. This heterogeneity, the substantial proportion of uncontrolled studies and the relatively small number of studies overall, should be borne in mind when considering our findings.

Overall, 15 of the 20 included studies reported improvements in appropriate prescription of OACs in eligible AF patients for stroke prevention, indicating that such inter- ventions can be effective in influencing HCPs' prescribing behaviours. Trials with a concurrent comparator were less likely to report significant improvements, raising questions about the appropriateness of uncontrolled study designs during the last decade, and strongly indicating the need for controlled studies to support appropriate conclusions. Studies with high stroke-risk AF patients $\left(\mathrm{CHA}_{2} \mathrm{DS}_{2}\right.$-VASc $\geq 2$ or according to guidelines) were less likely to show improvement in appropriate OAC prescription than those with participants at moderate-high or unspecified stroke risk. This finding may reflect the reported reluctance of HCPs to prescribe OACs to patients over 65 years who are likely to be at greater risk of stroke but also of falls and subsequent haemorrhage. ${ }^{9}$

Certain intervention designs appeared to be less effective in modifying HCPs' prescribing behaviours than others, such as computerized decision support tools and reviews of current prescribing. The integration of computerized decision support tools within electronic medical record systems is very common now; however, the greater the number of reminders or alerts a HCP receives the less likely they are to respond. ${ }^{44}$ This alert fatigue, ${ }^{44}$ may limit effectiveness of such interventions. Reviews of prescribing were often conducted by pharmacists, and it may be that clinicians respond better to interventions conducted by peer clinicians as discussed below.

Interventions based on HCP education, implementing guidelines or protocols and medical care programs were the most effective. The majority of HCP education and guideline/protocol implementation interventions had an element of persuasion in their theoretical focus (often facilitating HCP involvement in local guideline production or comparing HCPs' practice with peers). In contrast, a systematic overview of the theoretical foci of studies in HCP behavioural change reviews indicated that interventions with persuasive elements have proffered inconsistent results. However, some success has been reported by studies using local opinion leaders to promote evidence-based practice in HCPs. ${ }^{45}$ Most of the effective medical care programs, and HCP education and guideline/protocol implementation interventions were conducted by clinicians or multidisciplinary groups. It may be that interventions enabling clinicians to seek consensus with, and receive expert opinion from, their peer group are particularly effective in prompting behavioural change. These findings suggest that persuasive interventions based on HCP-peer group interaction, or both HCPs and patients in medical care programs, may be effective models for prompting behavioural change in OAC prescription for stroke prevention in eligible AF patients. These findings may also be useful for intervention design in other fields where HCP behavioural change is sought; however, large-scale cluster RCTs are required to determine the most effective HCP behaviour change interventions with greater certainty.

\section{Strengths}

This article represents the first systematic review of interventions to improve appropriate OAC prescription in 
eligible AF patients for stroke prevention. Despite the inclusion of only 20 studies, this research was based on 29,868 patients seen in 392 health care setting providing primary, secondary and tertiary care. This review was methodologically robust, with independent, duplicated screening, data extraction and quality assessment processes. Translation of non-English language papers guaranteed the inclusion of appropriate material. The main outcome was change in rate of OAC prescription to eligible AF patients for stroke prevention; rate of OAC use was only considered if the intervention was aimed only at HCPs. This distinction guaranteed that we explored the effect of the interventions directly on the HCP, not the patient. Additionally, the theoretical underpinnings of studies were examined to provide suggestions regarding the most effective elements, as recommended by the Medical Research Council. This approach is unique to a review in this field.

\section{Limitations}

Substantial heterogeneity in study design prohibited metaanalysis of individual results, preventing authors from drawing quantifiable conclusions regarding the effectiveness of interventions overall. This heterogeneity limits the certainty of conclusions drawn regarding the effectiveness of different study characteristics. Only 4 of the 20 included studies had concurrent controls, with the majority having either historical control or no control data, introducing a substantial risk of bias. This review included a comparatively small total number of studies $(n=20)$; however, the large total patient population (29,868 patients at baseline) and the consistency of many of the findings adds confidence to our observations. The lack of measures reporting the fidelity of the interventions to the intended method in the included studies creates uncertainty as to the effective elements within interventions, which should be addressed in the design of future studies. It should also be noted that the included studies provided very little consideration of the effect of their interventions on clinical outcomes, such as rates of stroke, haemorrhage and TIA. Future studies should include long-term follow-up of relevant clinical outcomes to improve their clinical relevance for both HCPs and patients.

\section{Conclusion}

AF is a growing global issue. Current research suggests that in many countries HCPs are still under-prescribing OACs in high stroke-risk patients and over-prescribing in low-risk patients. Effective interventions are needed to improve appropriate prescription of OACs in eligible AF patients to prevent unnecessary risk of stroke and haemorrhage. This review suggests that effective interventions should include persuasive elements delivered by HCPs to HCPs or multidisciplinary teams. HCP education, implementing guidelines/protocols and medical care programs with education for both HCPs and patients may all be effective interventions. These findings may also inform development of behavioural change interventions for HCPs in other health care fields.

\section{What is known about this topic?}

- AF is an increasing international health concern. AF affects over 33 million people worldwide with prevalence increasing globally. The growth of the aging population in developed countries is a substantial contributing factor.

- Poor global guideline adherence in OAC prescription. Despite recent improvements, greater guidelineadherence is needed worldwide in the prescription of OACs to eligible AF patients for stroke prevention. Under-prescription in higher stroke-risk patients, including those over 65 years, and substantial overprescription in those at low risk is still reported globally, putting patients at unnecessary risk of stroke and haemorrhage.

\section{What does this paper add?}

- The first review of interventions aimed at HCPs.

This is the first systematic review of interventions specifically designed to improve HCPs' appropriate prescription of OACs in eligible AF patients for stroke prevention.

- Effective interventions could improve guideline adherence, reducing stroke and haemorrhage.

This review provides an indication of which intervention designs and theoretical foci may be most effective. Effective, practical, behaviour change interventions could be readily integrated into health care systems and have the potential to increase appropriate $\mathrm{OAC}$ prescription in eligible AF patients for stroke prevention, reducing rates of avoidable stroke and haemorrhage.

\section{Authors' Contributions}

K.J., R.V.P., D.A.L., G.N.T., D.B. and J.L.C. conceived the review and developed the methodological strategy; D.B., N.T., R.V.P. and R.F. performed the study selection; R.V.P., D.B. and G.N.T. performed study selection, data extraction and quality assessment with K.J. and D.A.L. as arbitrators. R.V.P. drafted the paper with input and critical review from all authors.

\section{Funding}

This research was conducted at the University of Birmingham. This research was funded by the National Institute for Health Research (NIHR) Collaboration for Leadership in Applied Health Research and Care West Midlands (NIHR CLAHRC WM). The views expressed in this article are those of the author(s) and not necessarily those of the NHS, the NIHR or the Department of Health and Social Care. 


\section{Conflict of Interest}

K.J. reports grants from National Institute for Health Research (NIHR), outside the submitted work, during the conduct of the study. D.A.L. reports grants from Boehringer Ingelheim and Bristol-Myers-Squibb, and personal fees from Boehringer Ingelheim, Bristol-MyersSquibb, Bayer, Pfizer and Daiichi-Sankyo, outside the submitted work. R.V.P., G.N.T., D.B., J.L.C. and R.F. report no competing interests.

\section{References}

1 Lane DA, Skjøth F, Lip GYH, Larsen TB, Kotecha D. Temporal trends in incidence, prevalence, and mortality of atrial fibrillation in primary care. J Am Heart Assoc 2017;6(05):e005155

2 Chugh SS, Havmoeller R, Narayanan K, et al. Worldwide epidemiology of atrial fibrillation: a Global Burden of Disease 2010 Study. Circulation 2014;129(08):837-847

3 Lip GY, Nieuwlaat R, Pisters R, Lane DA, Crijns HJ. Refining clinical risk stratification for predicting stroke and thromboembolism in atrial fibrillation using a novel risk factor-based approach: the euro heart survey on atrial fibrillation. Chest 2010;137(02):263-272

4 National Institute for Health and Care Excellence (NICE). Atrial fibrillation: management: clinical guideline 180; 2014

5 Kirchhof P, Benussi S, Kotecha D, et al; ESC Scientific Document Group. 2016 ESC Guidelines for the management of atrial fibrillation developed in collaboration with EACTS. Eur Heart J 2016;37 (38):2893-2962

6 Pisters R, Lane DA, Nieuwlaat R, de Vos CB, Crijns HJ, Lip GY. A novel user-friendly score (HAS-BLED) to assess 1-year risk of major bleeding in patients with atrial fibrillation: the Euro Heart Survey. Chest 2010;138(05):1093-1100

7 Steinberg BA, Gao H, Shrader P, et al; GARFIELD-AF; ORBIT-AF Investigators. International trends in clinical characteristics and oral anticoagulation treatment for patients with atrial fibrillation: results from the GARFIELD-AF, ORBIT-AF I, and ORBIT-AF II registries. Am Heart J 2017;194:132-140

8 Proietti M, Laroche C, Opolski G, Maggioni AP, Boriani G, Lip GYH; AF Gen Pilot Investigators. 'Real-world' atrial fibrillation management in Europe: observations from the 2-year follow-up of the EURObservational Research Programme-Atrial Fibrillation General Registry Pilot Phase. Europace 2017;19(05):722-733

9 Lip GY, Laroche C, Dan GA, et al. 'Real-world' antithrombotic treatment in atrial fibrillation: the EORP-AF pilot survey. Am J Med 2014;127(06):519-29.e1

10 Pugh D, Pugh J, Mead GE. Attitudes of physicians regarding anticoagulation for atrial fibrillation: a systematic review. Age Ageing 2011;40(06):675-683

11 Vallakati A, Lewis WR. Underuse of anticoagulation in patients with atrial fibrillation. Postgrad Med 2016;128(02):191-200

12 Higgins J, Green S, Scholten R. Maintaining reviews: updates, amendments and feedback. In: Higgins J, Green S. Cochrane Handbook for Systematic Reviews of Interventions. Chichester, UK: John Wiley \& Sons; 2008:297-333

13 Moher D, Liberati A, Tetzlaff J, Altman DG; PRISMA Group. Preferred Reporting Items for Systematic Reviews and MetaAnalyses: the PRISMA statement. Int J Surg 2010;8(05):336-341

14 Johnson MJ, May CR. Promoting professional behaviour change in healthcare: what interventions work, and why? A theory-led overview of systematic reviews. BMJ Open 2015;5(09):e008592

15 The Cochrane Collaboration. Cochrane Handbook for Systematic Reviews of Interventions. Version 5.1.0 (updated March 2011). London, UK: The Cochrane Collaboration; 2011

16 National Heart Lung and Blood Institute. Quality assessment tool for observational cohort and cross-sectional studies; 2014 . Available at: http://www.nhlbi.nih.gov/health-pro/guidelines/in-develop/cardiovascular-risk-reduction/tools/cohort. Accessed February 6, 2018
17 Arts DL, Abu-Hanna A, Büller HR, Peters RJG, Eslami S, van Weert HCPM. Improving stroke prevention in patients with atrial fibrillation. Trials 2013;14:193

18 Lee TM, Ivers NM, Bhatia S, et al. Improving stroke prevention therapy for patients with atrial fibrillation in primary care: protocol for a pragmatic, cluster-randomized trial. Implement Sci 2016;11(01):159

19 Rao MP, Ciobanu AO, Lopes RD, et al. A clustered randomized trial to IMProve treatment with AntiCoagulanTs in patients with Atrial Fibrillation (IMPACT-AF): design and rationale. Am Heart J 2016; 176:107-113

20 Willis TA, Hartley S, Glidewell L, et al; ASPIRE programme. Action to Support Practices Implement Research Evidence (ASPIRE): protocol for a cluster-randomised evaluation of adaptable implementation packages targeting 'high impact' clinical practice recommendations in general practice. Implement Sci 2016;11:25

21 Bajorek BV, Magin PJ, Hilmer SN, Krass I. Optimizing stroke prevention in patients with atrial fibrillation: A cluster-randomized controlled trial of a computerized antithrombotic risk assessment tool in Australian general practice, 2012-2013. Prev Chronic Dis 2016;13:E90

22 Arts DL, Abu-Hanna A, Medlock SK, van Weert HC. Effectiveness and usage of a decision support system to improve stroke prevention in general practice: a cluster randomized controlled trial. PLoS One 2017;12(02):e0170974

23 Holt TA, Dalton A, Marshall T, et al. Automated software system to promote anticoagulation and reduce stroke risk: cluster-randomized controlled trial. Stroke 2017;48(03):787-790

24 Boriani G, Santini M, Lunati M, et al; Italian ClinicalService Project. Improving thromboprophylaxis using atrial fibrillation diagnostic capabilities in implantable cardioverter-defibrillators: the multicentre Italian ANGELS of AF Project. Circ Cardiovasc Qual Outcomes 2012;5(02):182-188

25 Touchette DR, Mcguinness ME, Stoner S, Shute D, Edwards JM, Ketchum K. Improving outpatient warfarin use for hospitalized patients with atrial fibrillation. Pharm Pract (Granada) 2008;6 (01):43-50

26 Hendriks JLM, Nieuwlaat R, Vrijhoef HJM, de Wit R, Crijns HJGM, Tieleman RG. Improving guideline adherence in the treatment of atrial fibrillation by implementing an integrated chronic care program. Neth Heart J 2010;18(10):471-477

27 Cook DA, Enders F, Caraballo PJ, Nishimura RA, Lloyd FJ. An automated clinical alert system for newly-diagnosed atrial fibrillation. PLoS One 2015;10(04):e0122153

28 Jackson SL, Peterson GM, Vial JH. A community-based educational intervention to improve antithrombotic drug use in atrial fibrillation. Ann Pharmacother 2004;38(11):1794-1799

29 Falces C, Andrea R, Heras M, et al. Integration between cardiology and primary care: impact on clinical practice [in Spanish]. Rev Esp Cardiol 2011;64(07):564-571

30 Wang Y, Bajorek B. Pilot of a Computerised Antithrombotic Risk Assessment Tool Version 2 (CARATV2.0) for stroke prevention in atrial fibrillation. Cardiol J 2017;24(02):176-187

31 Sobrequés J, Espinasa J, Cebrià J. Effectiveness of an intervention programme to improve oral anti-coagulation treatment for patients with chronic auricular fibrillation in a health district [in Spanish]. Aten Primaria 2002;30(09):588-589

32 Lowdon DW, Harper JR, Gillespie ND. Improving thromboprophylaxis in elderly patients with non-valvular atrial fibrillation. Scott Med J 2004;49(04):148-150

33 Bajorek BV, Krass I, Ogle SJ, Duguid MJ, Shenfield GM. Optimizing the use of antithrombotic therapy for atrial fibrillation in older people: a pharmacist-led multidisciplinary intervention. J Am Geriatr Soc 2005;53(11):1912-1920

34 Bo S, Valpreda S, Scaglione L, et al. Implementing hospital guidelines improves warfarin use in non-valvular atrial fibrillation: a before-after study. BMC Public Health 2007;7:203

35 Coll-Vinent B, Pacheco G, Junyent M, et al. Impact of implementing common guidelines at different care levels in a healthcare area 
on the improvement of atrial fibrillation treatment [in Spanish]. Rev Esp Cardiol 2007;60(04):392-403

36 Jackson SL, Peterson GM. Stroke risk assessment for atrial fibrillation: hospital-based stroke risk assessment and intervention program. J Clin Pharm Ther 2011;36(01):71-79

37 Oliveira R, Grilo S, Moreira C, et al. A quality study to improve prophylactic antithrombotic therapy prescribed to patients with atrial fibrillation. Rev Port Cardiol 2014;33(02):89-94

38 Robson J, Dostal I, Mathur R, et al. Improving anticoagulation in atrial fibrillation: observational study in three primary care trusts. Br J Gen Pract 2014;64(622):e275-e281

39 Das M, Panter L, Wynn GJ, et al. Primary Care Atrial Fibrillation Service: outcomes from consultant-led anticoagulation assessment clinics in the primary care setting in the UK. BMJ Open 2015; 5(12):e009267

40 Hsieh F-I, Jeng J-S, Chern C-M, et al; BTS-Stroke Investigators. Quality improvement in acute ischemic stroke care in Taiwan: the breakthrough collaborative in stroke. PLoS One 2016;11(08): e0160426

41 Guimarães PO, Wojdyla DM, Alexander JH, et al. Anticoagulation therapy and clinical outcomes in patients with recently diagnosed atrial fibrillation: insights from the ARISTOTLE trial. Int J Cardiol 2017;227:443-449

42 Clarkesmith DE, Pattison HM, Lane DA. Educational and behavioural interventions for anticoagulant therapy in patients with atrial fibrillation. Cochrane Database Syst Rev 2013;(06): CD008600

43 Clarkesmith DE, Pattison HM, Khaing PH, Lane DA. Educational and behavioural interventions for anticoagulant therapy in patients with atrial fibrillation. Cochrane Database Syst Rev 2017;4:CD008600

44 Ancker JS, Edwards A, Nosal S, Hauser D, Mauer E, Kaushal R; with the HITEC Investigators. Effects of workload, work complexity, and repeated alerts on alert fatigue in a clinical decision support system. BMC Med Inform Decis Mak 2017;17(01):36

45 Flodgren G, Parmelli E, Doumit G, et al. Local opinion leaders: effects on professional practice and health care outcomes. Cochrane Database Syst Rev 2011;(08):CD000125

46 Hankey GJ; National Blood Pressure Advisory Committee of the National Heart Foundation. Non-valvular atrial fibrillation and stroke prevention. Med J Aust 2001;174(05):234-239

47 Singer DE, Albers GW, Dalen JE, Go AS, Halperin JL, Manning WJ. Antithrombotic therapy in atrial fibrillation: the seventh ACCP conference on antithrombotic and thrombolytic therapy. Chest 2004;126(3, Suppl):429S-456S

48 Scottish Intercollegiate Guideline Network. Antithrombotic therapy. A national clinical guideline. Publication no. 36. Edinburgh;1999

49 Hart R, Benavente O. Primary prevention of stroke in patients with atrial fibrillation. In: Cobbe S, Royal College of Physicians of Edinburgh, eds. Atrial Fibrillation in Hospital and General Practice: The Sir James Mackenzie Consensus Conference. Edinburgh, United Kingdom1999

50 National Health and Medical Research Council. Prevention of Stroke: A Guide for General Practitioners. Canberra, Australia; 1997

51 Fuster V, Rydén LE, Cannom DS, et al; American College of Cardiology; American Heart Association Task Force; European Society of Cardiology Committee for Practice Guidelines; European Heart Rhythm Association; Heart Rhythm Society. ACC/AHA/ESC 2006 guidelines for the management of patients with atrial fibrillation: full text: a report of the American College of Cardiology/American Heart Association Task Force on practice guidelines and the European Society of Cardiology Committee for Practice Guidelines (Writing Committee to Revise the 2001 guidelines for the management of patients with atrial fibrillation) developed in collaboration with the European Heart Rhythm Association and the Heart Rhythm Society. Europace 2006;8(09):651-745 